\title{
Vision of Humanity humanistic value of the wenshan Miao Martial
}

\section{Arts Appreciation}

\author{
Qiaohenyan
}

Wenshan College

346591653@163.com

\author{
Keywords:Culture;Miao martial arts;Humanistic Value
}

\begin{abstract}
Miao is a worldwide ethnic,Hmong culture is the excellent Chinese cultural heritage.This article excavated from a cultural point of humanistic value of the Miao martial arts, and heritage and development carried out to provide a reference for the development of the Miao martial arts.
\end{abstract}

\section{Introduction}

Culture is the significance of the system people, man and nature, between people and ideas.Culture is considered the facts, inheritance and development, is the only channel for human communication.Wenshan Miao martial arts is not just a physical fitness movement, but the history of precipitation Hmong culture, Hmong ancestors wisdom.In the historical development of today can not be the Miao martial arts and cultural split off.The rapid development of a sport is inseparable from their cultural backgrounds, different cultural backgrounds, their development pattern will be different.Miao in the tradition of martial arts only inherited the essence of the process of the Miao culture in order to better carry forward the Miao martial arts.

The origin of the Miao martial arts. Wenshan Miao migration from the late Ming and early Qing Dynasty lasted until, according to "Maguan County" records, Wenshan Miao is the migration from the Ming and Qing Dynasties, the majority moved to the Guizhou Province. For the record Miao martial arts, about 1856 items Xichou from Miao Zhou was sent to military school, since then trained in a good martial arts. Now, the "Life of 迸 put" is recorded, Wenshan, Pingbian Hmong people love martial arts, martial arts called "Bukit security" in the Hmong. Hmong boxing and martial arts equipment is divided into two categories, the most famous is Lusheng boxing punches and hand fist, hand fist is unarmed, Lusheng Lusheng dance and boxing is boxing Miao together, not only has a strong attack and defense consciousness and also ornamental. Devices have knives, sticks, guns, axes, swords and other forms. Miao martial arts has a staunch temperament, steady pace, tactics varied features, but the Hmong teach martial arts in the process, great emphasis on Takenori education, teach skills prior to the master, apprentice training are to be sworn in before the Patriarch spirit of respect for teachers, Ming truth, not life and death can not be used martial arts to hurt others. Thus, among the Hmong people martial arts are numerous, but rarely stir up trouble, most martial arts are heavy duty, known etiquette, Ken patience.

With the social development and historical changes, National Fusion increasingly evident, the Miao is also undergoing profound changes, more and more people not only from the clothes change, but also from the spirit and soul has been greatly changed. More and more people accept the novelty, it is vulnerable to external cultural assimilation, and for the traditional Chinese martial arts Hmong lost its interest, so Miao martial arts gradually decline. 
The cultural value of the Miao Martial Arts. Miao is a worldwide nation. Classical, and there are records of Miao People of China as early as 5000 years ago, mostly living in today's Hunan, eastern Guizhou and other places, that is at the junction of Guizhou, Guangxi, Hainan, Hunan, Hubei, Sichuan, Yunnan and other places zone. Miao culture is known as the world's third special type of culture, ranging between nomadic and farming in the process of migration of Miao ancestors, their ethnic customs, habits, lifestyles constitute the main features of the Miao culture. Hmong Miao people due to the different places of residence and with differentiation between a long time because many Miao region and Han exchanges, most of the use of Chinese language as the main language. Miao have many processes, such as cross-stitch, batik, jewelry production, etc., which has thousands of years of history of batik, famous, more than one hundred and thirty kinds of clothing. Miao Miao's Flower Festival has a long history of tradition of important festivals, Miao folk culture of performance with outstanding representative Miao cultural unity of form and content, the combination of the Miao people spiritual strength.

Defensive Value. Miao martial arts is to produce the beginning of self-defense. Hmong original settlers in harsh environments by tenacious survival awareness and simple tools to hunt for food, to protect their own safety, in order to obtain the right to life, while preventing damage to foreign invaders. With the accumulation of life experience, Miao People gradually realized that summarize the experience of combat, thereby increasing the importance of the fight itself and defense capabilities. In the hunt for food according to their own, in order to defend the homeland combat, summarized some simple and practical method of self-defense, the selection of some of the young people to be better physical fitness exercises, this is the first Miao martial arts fighting shape. During the great migration of several Miao, Miao fought martial arts as an important means of ethnic people continue to survive. Miao martial arts development today, already it has both offensive and defensive characteristics, including the Miao policy in the hands of the "thirty-six attack, seventy anti."

Value Fitness. Initially the Miao martial arts for self-defense, to today, also has the effect of physical fitness [2]. Miao martial arts to learn the essence of health science, traditional medicine and other disciplines in the process of development, for the majority of the Miao people love. Unlike other martial arts fitness programs Hmong characterized by focusing internally and externally, body and spirit raising a total of practice concept, emphasizing the unity of consciousness and external, that the concept of body and mind. And externally, mainly from human organs and people's spiritual awareness of body posture colleagues combine, not only requires people to pay attention at the time of exercise of exercise, but also through spiritual practice, sense of control to achieve physical health, physical and mental balanced development. Other sports physical activity mostly dominated, then in the process of re-training to get psychological pleasure [3], and Miao martial arts to consciousness as a guide, starting from the heart and mind, consciousness, gas, access to psychological adjustment, from the inside out, and then making full body exercise to unwind and exercise. In today's society, people's health is the ideal goal pursued by the Miao martial arts exercises, can improve cardiovascular function and health, physical fitness to meet people's needs. Further improve so that more understanding of the Hmong culture, increase harmony between peoples, enrich people's spiritual and cultural life.

Humanistic spirit and educational value. Hmong Wushu and individuals and groups in two forms, of which the individual is the singles, doubles and collective groups divided. Content in various forms, there are unarmed, instruments and acupuncture. Unarmed into the basics, making hand Huaquan and skirting racks, seedlings boxing techniques, footwork and other multi-purpose Hmong named response characteristics Hmong language, such as "play called dig popcorn" (monkey 
breaking corn), "Gou move take out particular. "(Chi fist), etc., from the Miao people in the production and life Xingyi similar action name borrowed from. Devices with long instruments, short instruments, soft instruments, etc., such as a knife, sword, meteor hammer, bamboo dart, hook knife, club and smoke. In addition there are qigong Miao martial arts, including hard power and soft power, hard power is mainly used for bruises, fractures and other clinical, soft power is mainly used for fever, bleeding and so on. Miao martial arts can not only increase the fighting skills, but also for physical fitness, the Hmong have been handed down in historical records can be seen, Takenori Hmong-speaking nation, recruit apprentice chef at the "three religions do not teach three, three-three not to fight, "the sect commandments. The so-called three no's three religions teach that bad temper do not teach, teach good temper; no idle Services We do not teach the positive industry sub honest person honest religion; disowned desperadoes do not teach, teach by heavy loyalty formality. The so-called three-three not to fight, that is, when life and death, for the culprit who must fight me, for those who do not play no intention to hurt me; my family who must fight against bullying, to be my beat and cymbals to beg me not to fight; to who must fight the first accident, the innocent do not play [1]. Shouyi's master at all times in the Spirit swear before Patriarch subject teacher training, especially for this type of acupuncture to take people between life and the hands, non-life and death to people absolutely can not be used.

Competitive and performances Value. Miao martial arts forms, rich in content, including unarmed forms of exercise, equipment, etc., in different festivals in Hmong performing different contents, Huashan Festival holiday to commemorate the legend Chi held children, using climbing form of flower stem to perform, most make use inverted climb, climb the highest is the winner who, along with traditional martial arts performances. Lusheng boxing is the use of a performance in the form of a combination of Lusheng and boxing, martial arts, both staunch lean, soft dance there will be an effective combination of strength and beauty, so that people in the process of watching martial arts fun to mold and sublimation. Therefore, Miao martial arts has pleasing aesthetic function Yue Chi, the aesthetic form encompasses three levels including mutual play their respective roles in the progression. Pleasing to the eye from the physical point of view, but also beyond physical sensory pleasure; Wyatt heart is in understanding, foster human emotion on mind and imagination aesthetic sense; Wyatt aim to achieve a life of emotional state on a moral basis, it is the concept of morality pursuit and satisfaction.

Cultural entertainment and recreational values. Traditional Sports of Minority activities are generally held during national holidays or seasonal, closely linked to the nation's productive activities or leisure activities [4]. The main festival of Miao Miao New Year and Spring Festival, Miao festivals prevalent in Guangxi and Guizhou, usually after Millet Jincang, mutual visits, singing and dancing, young men and women and other mutual antiphonal singing, lasted about nine days to end; Miao and Han Spring Festival time, as different regions with different Miao celebration, such as cattle drive, jump Lusheng dance, bullfighting and other express joy Xiang Teng wishes. Flower Festival is a traditional festival of the Miao, various regions are not the same time, the Flower Festival of the Miao people in the first month, usually held pole climbing flowers, Lusheng dance and other activities, the elderly also took the opportunity to visit family, young men and women to each other Duige expressing affection. The traditional Dragon Boat Festival holiday there, catch-Autumn Festival, these festivals are on or spare at the end of the busy expressing longing for a harmonious, healthy and happy life, which is currently our people want to achieve. With increasingly fierce competition, people's increasing pressure, most of the city's people are yearning for paradise place, and Yunnan scenic meandering, like spring, it is a good place to visit to relax. In the small holiday and the Spring Festival, many people take time out from major cities to the town, 
to the village, blows the pressure of work, to find the true self in the Miao people's festival, feel the Hmong culture, appreciation of the Miao dance, into the large Hmong family, involved in the activities of the Hmong, to improve their physical and mental health.

\section{Reference}

[1] Pinhua Zhang Wenshan Miao Wushu Present Situation Investigation and Development Countermeasure Research [D] Capital Institute of Physical Education, 2011,5: 10-18.

[2] Heqiong wen.Changes in social structure village Inheritance and Protection of Minority Nationalities Sports in [J] Jiamusi Education Institute, 2013,12 (134): 427-428.

[3] Honggang yuan.To investigate the value and role of traditional sports of ethnic minorities in Yunnan [J] Contemporary Sports Science, 2011,1 (1): 73-75.

[4] Bingnan zhao."humanism" and the humanistic value of traditional national sport interpretation of [J] Physical Education, 2014,14 (4): 56-57. 